\title{
ChemComm
}

\section{Studies of G-quadruplexes formed within self-assembled DNA mini-circles $\dagger$}

Cite this: Chem. Commun., 2016, 52,12454

Received 30th August 2016, Accepted 22nd September 2016

DOI: $10.1039 / c 6 c c 07110 d$

www.rsc.org/chemcomm

We have developed self-assembled DNA mini-circles that contain a G-quadruplex-forming sequence from the $c-M y c$ oncogene promoter and demonstrate by FRET that the G-quadruplex unfolding kinetics are 10-fold slower than for the simpler 24-mer G-quadruplex that is commonly used for FRET experiments.

In the presence of monovalent cations such as $\mathrm{Na}^{+}$and $\mathrm{K}^{+}$, guanine-rich sequences of DNA can fold into quadruply-stranded helical structures known as G-quadruplexes. The sequences that give rise to these structures are prevalent in genomic DNA, and there is experimental evidence for a role of G-quadruplexes in the regulation of gene expression, translation, telomere-end maintenance, recombination and replication. ${ }^{1-5}$ In vitro biophysical and spectroscopic studies of G-quadruplex-forming sequences can contribute significantly to understanding how their intrinsic properties relate to their diverse biological functions. Such studies include characterization by UV/Vis and circular dichroism (CD) spectroscopies, fluorescence resonance energy transfer (FRET) studies, NMR spectroscopy, surface plasmon resonance (SPR) and calorimetric techniques. However, such studies are normally carried out using short oligonucleotides as models for the sequences of interest (Fig. 1c; G4-24-mer). This is a fundamental problem since, in cells, G-quadruplex DNA motifs are not isolated short oligos, but are flanked by long

\footnotetext{
${ }^{a}$ Department of Chemistry, Imperial College London, London, SW7 2AZ, UK. E-mail: r.vilar@imperial.ac.uk

${ }^{b}$ Institute of Chemical Biology, Imperial College London, London, SW7 2AZ, UK. E-mail: l.ying@imperial.ac.uk

${ }^{c}$ London Centre for Nanotechnology, University College London, London, WC1H OAH, UK

${ }^{d}$ Department of Physics and Astronomy, University College London, London, WC1E 6BT, UK

${ }^{e}$ Molecular Medicine, National Heart and Lung Institute, Imperial College London, London, $S W 72 A Z, U K$

$\dagger$ Electronic supplementary information (ESI) available: Experimental details including preparation and purification of mini-circles, atomic force microscopy (AFM) of mini-circles and single molecule FRET studies. This data is openly available. See DOI: 10.1039/c6cc07110d

\# These three authors contributed equally to the work.
}

(a)

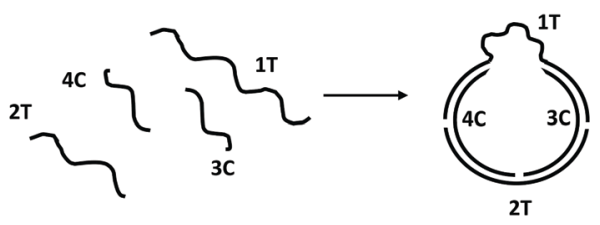

(b)

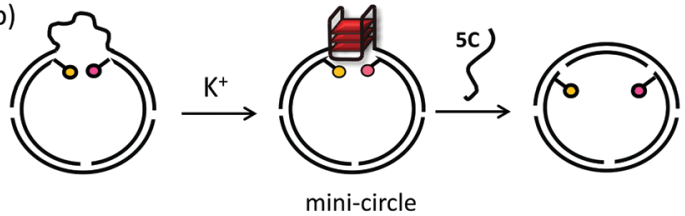

(c)

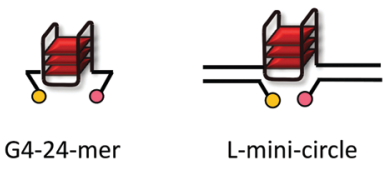

Fig. 1 Schematic representation of systems under study. (a) DNA mini-circles assembled from 1T, 2T, 3C and 4C single stranded oligos (see Table S1 in the $\mathrm{ESI} \dagger$ for details of the sequences). $1 T$ contains the G-quadruplex forming sequence Myc-2345. (b) For FRET studies 3C and 4C strands were labelled with FAM and TAMRA respectively. G-quadruplex unfolding can be studied by its hybridisation to complementary strand $5 \mathrm{C}$ leading to decrease in FRET. (c) G-quadruplex folded from a 24-mer (referred to as G4-24-mer; left); linearised half mini-circle (referred to as L-mini-circle; right).

stretches of duplex DNA, and packed in the genome. This restricts the topology of the G-quadruplex containing DNA motifs and generally leads to supercoiled DNA conformations. In particular, during folding of naturally occurring G-quadruplexes, their $5^{\prime}$ - and $3^{\prime}$-ends would have fewer degrees of freedom compared to short single-stranded oligos that are currently used for in vitro model studies. Thus, these short DNA sequences can fold into several different G-quadruplex topologies that might not be biologically relevant. ${ }^{6}$ At present, it is unclear to what extent G-quadruplex formation is affected by flanking DNA and topological restrictions. Therefore, there is a need for novel models of G-quadruplexes that are more representative of the native cellular environment (e.g. where additional restrictions are imposed on the $3^{\prime}$ and $5^{\prime}$ ends of 
the G-quadruplex). Recently, several strategies have been reported to address the above issues. For example, G-quadruplexes have been embedded in frameworks of larger oligonucleotides, such as DNA origami, ${ }^{7,8}$ or their ends have been mechanically constrained by attaching them to beads for studies using optical ${ }^{9-12}$ and magnetic ${ }^{13-15}$ tweezers. However, these models are not readily accessible, or amenable for developing high-throughput screening systems. Here we present self-assembled DNA mini-circles (Fig. 1a and b) as novel tools to study G-quadruplex unfolding kinetics. This system constrains the $5^{\prime}$ and $3^{\prime}$ ends of the G-quadruplex forming sequence to more closely resemble their in vivo environment, can be optically labelled for high-throughput FRET studies, and can be readily assembled from five short oligonucleotides. To validate our new model, we have compared the unfolding kinetics of G-quadruplexes in these mini-circles with a simple 24-mer G-quadruplex (G4-24-mer) typically used for FRET melting experiments (Fig. 1c; see Table S1 for sequences, ESI $\dagger$ ) as well as with a linearised version of the mini-circle (L-mini-circle, Fig. 1c; see Table $\mathrm{S} 1$ for sequences, ESI $\dagger$ ).

A number of previous studies have shown that DNA minicircles can be assembled from short oligonucleotides ${ }^{16-19}$ and that they can be used to study the topological properties of nucleic acids. ${ }^{19-23}$ For our studies we chose a well characterised G-quadruplex DNA structure from the promoter of $c-M y c$, namely the four G-tract sequence Myc-2345 (see 1T sequence in Table S1, $\mathrm{ESI} \dagger$ ). Previous studies have shown that this sequence can form stable G-quadruplexes under physiological conditions and it has been proposed that they play an important role in gene regulation. ${ }^{24}$

The mini-circles containing c-Myc-2345 were assembled by annealing the four single-stranded DNA oligonucleotides 1T, 2T, 3C and 4C as shown in Fig. 1. To facilitate FRET studies, donor (FAM) and acceptor (TAMRA) fluorophores were positioned at the $3^{\prime}$ end of the $3 \mathrm{C}$ sequence and at the $5^{\prime}$ end of the $4 \mathrm{C}$ sequence respectively (see Table S1 in the ESI $\dagger$ ). Depending on the conformation, G-quadruplex folding would result in a separation between the donor/acceptor fluorophore pair of 11 to 16 bases leading to efficient FRET. On the other hand, when the structure is unfolded, the separation between the fluorophores would be 32 bases (which corresponds to $c a .11 \mathrm{~nm}$ ), leading to a significant decrease in FRET efficiency which can be readily monitored by fluorescence spectroscopy. Such a design should be optimal to study the unfolding kinetics of the G-quadruplex structure.

The mini-circles were assembled by annealing equimolar amounts of the four strands $1 \mathrm{~T}, 2 \mathrm{~T}, 3 \mathrm{C}$ and $4 \mathrm{C}$ in the presence of $\mathrm{Li}^{+}$ions (and in the presence of $\mathrm{Na}^{+}$in the case of the FAM/ TAMRA labelled mini-circles). Native PAGE of this mixture showed several bands (lane 2 in Fig. S4, ESI $\dagger$ ) suggesting the formation of several DNA assemblies, however the desired mini-circles can be readily separated and purified (lane 3 in Fig. S4, ESI $\dagger$ ). Despite the lack of ligation, the self-assembled mini-circles were stable thermodynamically, as no aggregation into larger structures was observed even after their storage in $\mathrm{NaCl}$ buffer at $4{ }^{\circ} \mathrm{C}$ for over three months.

The PAGE-purified mini-circles were studied by atomic force microscopy (AFM) ${ }^{25}$ which showed DNA loops with contour lengths in the rather narrow range between $c a .41$ and $46 \mathrm{~nm}$ (Fig. 2A).
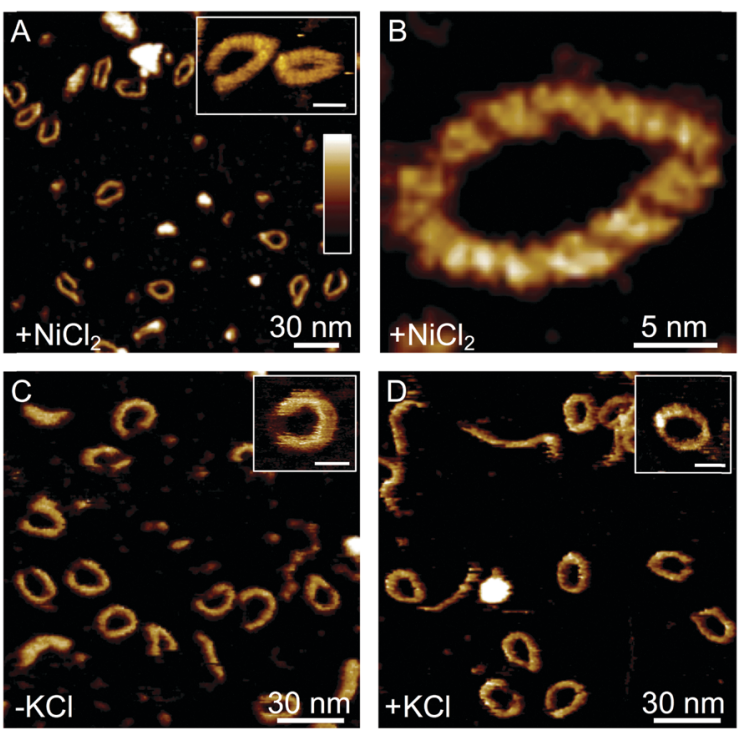

Fig. 2 Characterisation of DNA mini-circles by atomic force microscopy in liquid. The topographic images show (A) a mix of mini-circle conformations immobilised on a mica substrate using $\mathrm{NiCl}_{2}$, (B) high resolution of the mini-circle secondary structure, (C) the 'open' mini-circle conformation immobilised without $\mathrm{KCl}$ on a mica substrate functionalised with a cationic polymer, (D) the 'closed' conformation immobilised on a mica substrate functionalised with a cationic polymer, with $100 \mathrm{mM} \mathrm{KCl}$. Insets in A, B, C show higher resolution scans of these conformations, scale bars in all insets are $10 \mathrm{~nm}$. Colour scales (see inset in (A)) for A, B, C and D are; $2 \mathrm{~nm}, 2 \mathrm{~nm}$, $2.5 \mathrm{~nm}, 2.5 \mathrm{~nm}$ respectively.

This is consistent with the expected length range for the minicircles schematically depicted in Fig. 1, with a predicted $39 \mathrm{~nm}$ contour length for the 116bp of double-stranded DNA (assuming a B-DNA conformation, $0.34 \mathrm{~nm}$ per $\mathrm{bp}$ ), and with an additional, variable and folding-dependent length for the remaining 21 unpaired bases in the construct. For comparison, $137 \mathrm{bp}$ $(116+21 \mathrm{bp})$ of double-stranded B-DNA would yield a contour length of $47 \mathrm{~nm}$. Higher-resolution images (Fig. 2B - see also Fig. S7 in $\mathrm{ESI} \dagger$ ) allow a tentative estimate of 11-12 turns of the double helix, again consistent with the prediction of 11 turns for 116bp of B-DNA (assuming 10.5bp per turn).

The mini-circles were most straightforwardly prepared and imaged on the AFM substrate in the presence of $\mathrm{Ni}^{2+}$ (Fig. 2A and B). ${ }^{25}$ This resulted in a variety of mini-circle shapes, including closed rings with a single protrusion - indicative of G-quadruplex formation - and apparently open, horseshoe shapes that on further inspection appeared to be closed by a short segment of $\sim 1 \mathrm{~nm}$ height, presumably unfolded single-stranded DNA (Fig. 2A and Fig. S9, ESI $\dagger$ ). To allow for a more systematic study of the effect of salts on G-quadruplex formation in the AFM experiments, we used DNA adsorption protocols with cationic surface functionalization (see ESI, $\dagger$ and Fig. S8). These did not require the presence of salts in the adsorption/imaging buffers. In the absence of salts, the "open" mini-circle configurations were prevalent (Fig. 2C and Fig. S8, ESI $\dagger$ ), whereas in the presence of $\mathrm{K}^{+}$(Fig. 2D and Fig. S8, ESI $\dagger$ ) the closed configuration dominated, often showing single protrusions that we interpret as signatures of G-quadruplex formation. 
The FAM/TAMRA labelled mini-circles were then used to study the unfolding kinetics of the G-quadruplex by FRET. We were first interested in studying the effect of different ions on the kinetic stability of the G-quadruplexes formed in the minicircles. These experiments were carried out in the presence of a 100 -fold excess of a strand (5C - see Table S1, ESI $\dagger$ ) that was complementary to the quadruplex-forming sequence Myc-2345, in a Tris-HCl buffer, $\mathrm{pH}$ 7.4. In the presence of $\mathrm{Li}^{+}$, no G-quadruplex structure is expected to form. Fully consistent with this expectation, FRET showed (Fig. 3a) no change in FAM emission after addition of the complementary strand, which suggests that the G-quadruplex forming sequence was already unfolded prior to adding the complementary strand 5C. On the other hand, in the presence of $\mathrm{Na}^{+}$ions, a G-quadruplex is expected to form. This was indeed observed via FAM/TAMRA efficient FRET: upon addition of the complementary strand, the G-quadruplex unfolded rapidly, as indicated by the rapid increase and then saturation of the fluorescence signal associated to FAM. This increase in emission can be explained by the loss of FRET between the fluorophores upon unfolding of the G-quadruplex by addition of the complementary strand (Fig. 3b). On the other hand, as expected, $\mathrm{K}^{+}$ions stabilised the G-quadruplex: in a solution of $1 \mathrm{mM} \mathrm{K}^{+}$and $99 \mathrm{mM} \mathrm{Na}^{+}$ions, a slower unfolding was observed with a $\tau_{\mathrm{D}}$ of $1408 \mathrm{~s}$ (Fig. 3c). This value was obtained from fitting the data to a mono-exponential decay (see ESI $\dagger$ for details). At a higher $\mathrm{K}^{+}$concentration, $40 \mathrm{mM} \mathrm{K}^{+}$ions (and $60 \mathrm{mM}$ $\mathrm{Li}^{+}$), there was no unfolding detected up to $1 \mathrm{~h}$ (Fig. 3d). This confirms the high stability of the $c-M y c$ G-quadruplex in the presence of this concentration of $\mathrm{K}^{+}$ions.

We then compared the difference (using FRET) in unfolding kinetics between the mini-circles and the G-quadruplex formed with the short oligonucleotide sequence G4-24-mer (Fig. 1c). In addition, we also carried out unfolding kinetic experiments with the linearised half mini-circle, i.e. L-mini-circle (Fig. 1c see Fig. S10 and S11 for AFM characterisation of this assembly, ESI $\dagger$ ). For all these experiments, we used the FAM/TAMRA
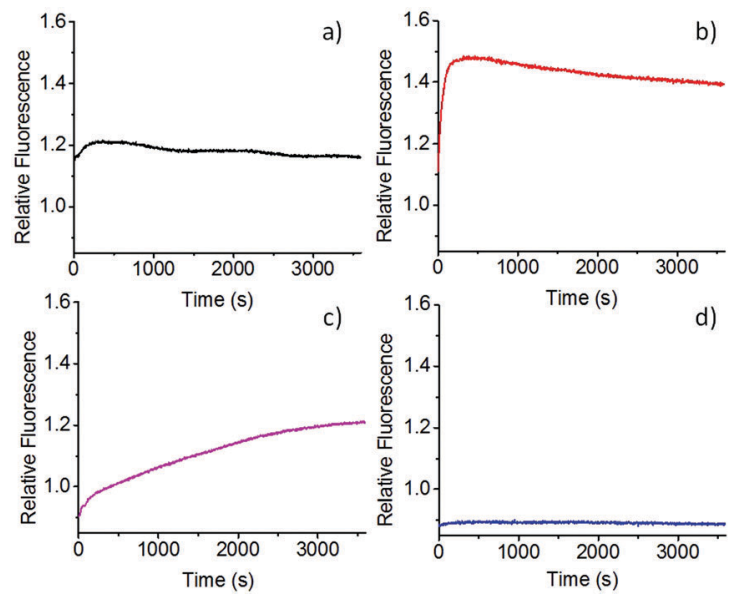

Fig. 3 Unfolding kinetics for the FAM/TAMRA-labelled mini-circles in the presence of (a) $\mathrm{Li}^{+}(100 \mathrm{mM})$, (b) $\mathrm{Na}^{+}(100 \mathrm{mM})$, (c) $\mathrm{K}^{+}(1 \mathrm{mM})+\mathrm{Na}^{+}$ $(99 \mathrm{mM})$ and $(\mathrm{d}) \mathrm{K}^{+}(40 \mathrm{mM})+\mathrm{Li}^{+}(60 \mathrm{mM})$ ions upon addition of the complementary strand $5 \mathrm{C}$. Curves shown are average of three repeats. labelled oligos and $99 \mathrm{mM} \mathrm{Na}{ }^{+} / 1 \mathrm{mM} \mathrm{K}^{+}$ions Tris-buffered solutions. The exponential decay curves were modelled with a mono-exponential or a bi-exponential function for the mini-circles/L-mini-circles and the G4-24-mer, respectively, to obtain a value of decay constant, $\tau_{\mathrm{D}}$ (see ESI $\dagger$ for details). The commonly used G4-24-mer G-quadruplex unfolds far more rapidly $\left(\tau_{\mathrm{D}}=120 \pm 43 \mathrm{~s}\right)$ than G-quadruplexes in mini-circles $\left(\tau_{\mathrm{D}}=1408 \pm 98 \mathrm{~s}\right)$, clearly demonstrating that the lower degree of conformational freedom one would expect in cells is an important factor to consider for in vitro models. In support of this rationale, the linearised mini-circles, which should have more degrees of freedom than the cyclised mini-circle but less than G4-24-mer, unfold at an intermediate rate between the two $\left(\tau_{\mathrm{D}}=1182 \pm 84 \mathrm{~s}\right)$.

Finally, we investigated the effect of the well-known G-quadruplex ligand pyridostatin (PDS) ${ }^{26}$ on the G-quadruplex unfolding kinetics of the three systems. Addition of a large excess of PDS (40 equivalents) to the three systems stabilised the G-quadruplex to such a high extent that no unfolding could be detected by FRET up to $3600 \mathrm{~s}$. When the excess was reduced to only 5 equivalents, upon addition of the complementary strand 5C, significantly slower unfolding of the G-quadruplex structure was observed in all three systems due to ligand binding (Fig. 4 bottom). The stabilising effect of PDS appeared largest on the G4-24-mer (ca. 10-fold increase in $\tau_{\mathrm{D}}$ compared to only $c a .1 .4$ fold for the minicircle), as indicated by the slower increase fluorescence over time relative to the control where no PDS was added. This may be due to the less hindered (and topologically more flexible) G-quadruplex structure formed by the short oligo compared to the mini-circle and its linearised analogue. In addition, unlike the mini-circle and L-mini-circle, the G4-24-mer G-quadruplex is not flanked by duplex DNA to which the PDS could bind.

In summary, we have shown that stable self-assembled DNA mini-circles with G-quadruplex motifs can be easily prepared, purified and used in FRET studies. We have evaluated unfolding kinetics of the $c-M y c$ G-quadruplex structure in three different systems and in the presence of different metal cations. These studies have shown that G-quadruplexes assembled from short sequences (such as G4-24-mer), which are used in most biophysical studies to characterize quadruplexes to date, display very different unfolding kinetics compared to a G-quadruplex embedded in a mini-circle. This is important given that native G-quadruplex sequences will be flanked by duplex regions like those present in the mini-circles. As the AFM studies showed (Fig. S10 and S11, ESI $\dagger$ ) the linearised version of the mini-circles is far less structured than the mini-circles where the G-quadruplex is clearly part of a more rigid duplex DNA framework (more alike to the environment this structure would be found in genomic DNA). Therefore, the mini-circle assembly provides a better mimic of genomic DNA than the unstructured linearised version. Finally, we have shown that PDS provides kinetic stability to the G-quadruplex within the mini-circles; it is important to highlight that since the G-quadruplex in the mini-circle is embedded in a duplex DNA framework, the conditions under which PDS binds to the G-quadruplex in this system are more alike to what would be encountered in a cellular environment. We therefore propose 

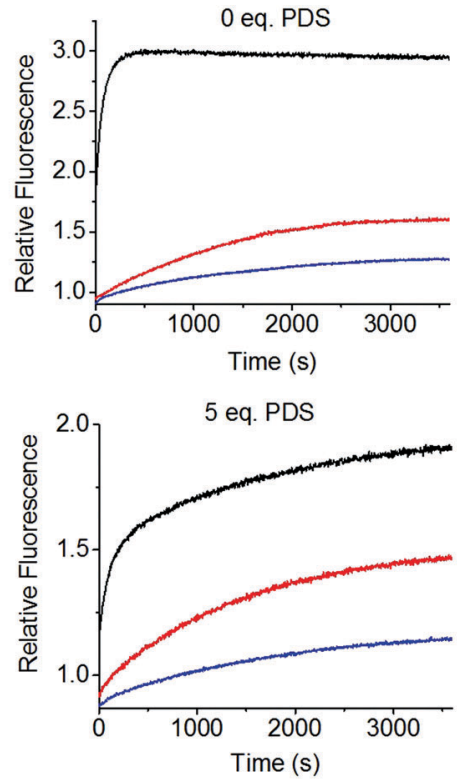

\begin{tabular}{ccc}
\hline System & $\boldsymbol{\tau}_{\mathrm{D}}$ (s) (0 eq. PDS) & $\boldsymbol{\tau}_{\mathrm{D}}$ (5 eq. PDS) \\
\hline Mini-circle & $1408 \pm 98$ & $1732 \pm 26$ \\
L-mini-circle & $1182 \pm 84$ & $1484 \pm 33$ \\
G4-24-mer & $120 \pm 43$ & $1376 \pm 97$
\end{tabular}

Fig. 4 Unfolding kinetics for the three systems under study (mini-circles (blue), 24-mer-G4 (black) and L-mini-circles (red)) in $99 \mathrm{mM} \mathrm{Na}^{+} / 1 \mathrm{mM} \mathrm{K}^{+}$ ions Tris-buffered solutions upon addition of the complementary strand $5 \mathrm{C}$ in the absence (top) and presence (bottom) of pyridostatin (PDS). Representative curves for each experiment have been presented. All experiments were performed in triplicate to obtain the error limits presented in the table.

G-quadruplex DNA mini-circles as a new platform to study the interaction of small molecule ligands (and potentially also proteins such as transcription factors) under conditions that are biologically more relevant than the simpler short-oligo G-quadruplexes widely used in most biophysical studies reported to date. We recognise that the self-assembled mini-circles herein reported are nicked and that this might have an effect on the folding/unfolding kinetics of the embedded G-quadruplexes. Therefore, future studies will focus on ligating the mini-circles and studying the effects that the increased rigidity of the assembly has on G-quadruplex formation.

The UK's Engineering and Physical Sciences Research Council (EPSRC) is thanked for financial support including a studentship to A. S. and B. K., fellowships to A. P. (EP/M506448/1) and R. V. (EP/H005285/1), and an equipment grant to B. W. H. (EP/M028100/1). Bruker (Santa Barbara, CA, USA) is thanked for technical support (particularly from Andrea Slade, Shuiqing $\mathrm{Hu}$ and Chanmin $\mathrm{Su}$ ) and for providing instrumentation.

\section{Notes and references}

1 S. Balasubramanian, Bioorg. Med. Chem., 2014, 22, 4356-4370.

2 B. P. Belotserkovskii, S. M. Mirkin and P. C. Hanawalt, Chem. Rev., 2013, 113, 8620-8637.

3 N. Maizels and L. T. Gray, PLoS Genet., 2013, 9, e1003468.

4 P. Murat and S. Balasubramanian, Curr. Opin. Genet. Dev., 2014, 25, 22-29.

5 S. Neidle, FEBS J., 2010, 277, 1118-1125.

6 G. N. Parkinson, Quadruplex Nucleic Acids, 2006, 1-30.

7 A. Rajendran, M. Endo, K. Hidaka and H. Sugiyama, Angew. Chem., Int. Ed., 2014, 53, 4107-4112.

8 A. Rajendran, M. Endo, K. Hidaka, P. L. T. Tran, J.-L. Mergny and H. Sugiyama, Nucleic Acids Res., 2013, 41, 8738-8747.

9 M. de Messieres, J.-C. Chang, B. Brawn-Cinani and A. La Porta, Phys. Rev. Lett., 2012, 109, 058101/058101.

10 D. Koirala, S. Dhakal, B. Ashbridge, Y. Sannohe, R. Rodriguez, H. Sugiyama, S. Balasubramanian and H. Mao, Nat. Chem., 2011, 3, 782-787.

11 Z. Yu, V. Gaerig, Y. Cui, H. J. Kang, V. Gokhale, Y. Zhao, L. H. Hurley and H. Mao, J. Am. Chem. Soc., 2012, 134, 5157-5164.

12 D. Koirala, T. Mashimo, Y. Sannohe, Z. Yu, H. Mao and H. Sugiyama, Chem. Commun., 2012, 48, 2006-2008.

13 S. Selvam, D. Koirala, Z. Yu and H. Mao, J. Am. Chem. Soc., 2014, 136, 13967-13970.

14 W. Li, X.-M. Hou, P.-Y. Wang, X.-G. Xi and M. Li, J. Am. Chem. Soc., 2013, 135, 6423-6426.

15 X. Long, W. Parks Joseph, R. Bagshaw Clive and D. S. Michael, Nucleic Acids Res., 2013, 41, 2746-2755.

16 W. Han, M. Dlakic, Y. J. Zhu, S. M. Lindsay and R. E. Harrington, Proc. Natl. Acad. Sci. U. S. A., 1997, 94, 10565-10570.

17 W. Han, S. M. Lindsay, M. Dlakic and R. E. Harrington, Nature, 1997, 386, 563 .

18 L. Ulanovsky, M. Bodner, E. N. Trifonov and M. Choder, Proc. Natl. Acad. Sci. U. S. A., 1986, 83, 862-866.

19 G. Rasched, D. Ackermann, T. L. Schmidt, P. Broekmann, A. Heckel and M. Famulok, Angew. Chem., Int. Ed., 2008, 47, 967-970.

20 A. D. Bates, A. Noy, M. M. Piperakis, S. A. Harris and A. Maxwell, Biochem. Soc. Trans., 2013, 41, 565-570.

21 Q. Du, M. Vologodskaia, H. Kuhn, M. Frank-Kamenetskii and A. Vologodskii, Biophys. J., 2005, 88, 4137-4145.

22 D. P. N. Goncalves, T. L. Schmidt, M. B. Koeppel and A. Heckel, Small, 2010, 6, 1347-1352.

23 T. Li and M. Famulok, J. Am. Chem. Soc., 2013, 135, 1593-1599.

24 A. Siddiqui-Jain, C. L. Grand, D. J. Bearss and L. H. Hurley, Proc. Natl. Acad. Sci. U. S. A., 2002, 99, 11593-11598.

25 A. Pyne, R. Thompson, C. Leung, D. Roy and B. W. Hoogenboom, Small, 2014, 10, 3257-3261.

26 R. Rodriguez, S. Muller, J. A. Yeoman, C. Trentesaux, J.-F. Riou and S. Balasubramanian, J. Am. Chem. Soc., 2008, 130, 15758-15759. 